\title{
$+3$ \\ CHARGED PARTICLE ENERGIZATION BY AN ARBITRARILY MOVING MAGNET
}

\section{T. J. BIRMINGHAM}

T. G. NORTHROP

GPO PRICE

s
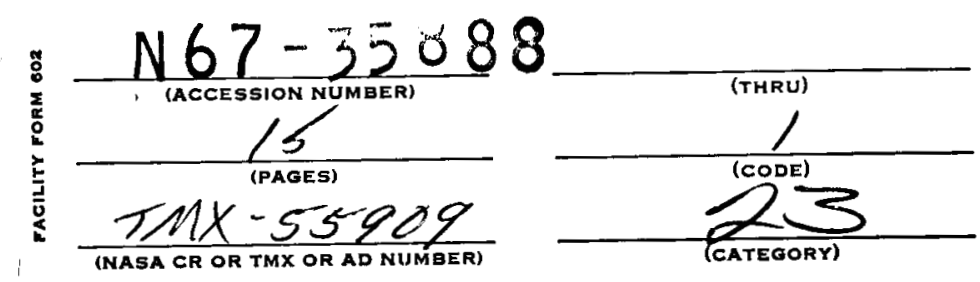

CFSTI PRICE(S)

Hard copy (HC)

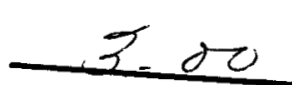

AUGUST 1967

Microfiche (MF)

ff 653 July 65

NASA

GODDARD SPACE FLIGHT CENTER

GREENBELT, MARYLAND

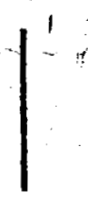


$X-641-67-404$

\section{CHARGED PARTICLE ENERGIZATION}

BY AN ARBITRARILY MOVING MAGNET

T. J. Birmingham

T. G. Northrop

AUGUST 1967

GODDARD SPACE FLIGHT CENTER

Greenbelt, Maryland 


\begin{abstract}
Second adiabatic theory is used to derive an asymptotic expression for the bounce-averaged rate of kinetic energy change $\langle\dot{w}\rangle$ experienced by charged particles trapped in electric and magnetic fields which conserve the first two invariants $M$ and $J$ of their motion. The restriction that there exist no electric field $E_{\|}$, parallel to the magnetic field, reduces $\langle\dot{W}\rangle$ by one order in the adiabatic expansion parameter $\mathrm{m} / \mathrm{e}$ from its $\mathrm{E}_{\|} \neq 0$ value. If in addition the magnetic field arises solely from, and convects rigidly with, the arbitrary motion of a perfectly conducting magnet, we find that $\langle\dot{W}\rangle$ is smaller by yet another order in $\mathrm{m} / \mathrm{e}$. Our results generalize and confirm the conclusions of Hones and Bergeson $[1965]$ who consider $E_{\|}=0$ and the special case of a uniformly rotating conducting magnetized sphere with non-aligned rotation and magnetization axes.
\end{abstract}




\section{CHARGED PARTICLE ENERGIZATION}

\section{BY AN ARBITRARILY MOVING MAGNET}

\section{INTRODUCTION}

Electric fields arising from the uniform rotation of a magnetized conducting sphere have been previously examined as a mechanism for the energization of charged particles. [Terletzky, 1946; Davis, 1947, 1948; Hones and Bergeson, 1965.] These studies have attempted to determine whether such electric fields (assuming that a star, the sun, or the earth can be modeled as such a sphere) can accelerate particles to cosmic ray and auroral energies or explain the high energy populations of planetary magnetospheres.

Cases where the rotation and magnetic axes are parallel [Davis, 1947, 1948] and arbitrarily aligned [ $\underline{\text { Terletzky }}, 1946$; Hones and Bergeson, 1965] have both been investigated. If the sphere is assumed to be surrounded by a vacuum, large accelerating voltages $\left(>10^{4}\right.$ volts for the earth and higher for the sun and stars) have been shown to exist along dipole field lines.

For such a sphere embedded in a plasma medium, however, the rotation induced electric field component $E_{\|}$parallel to the exterior dipole lines can be cancelled to a large extent by an electrostatic field arising from small plasma charge separations along the field lines. Assuming that $E_{\|}=0$ exactly, Hones and Bergeson $[1965]$ have shown that $\langle\dot{W}\rangle /$ e for trapped particles vanishes through second order in $\epsilon \equiv \mathrm{m} / \mathrm{e}$ : in the magnetic field of a dipole rotating with 
magnetization and rotation vectors arbitrarily aligned, trapped particles lose as much energy over one half of their bounce motion as they gain over the other half if $\mathrm{E}_{\|}=0$ 。

In this paper we first derive from second adiabatic theory [Northrop, 1963] an expression for the $\langle\dot{W}\rangle$ of a trapped particle. The leading order contribution to $\langle\dot{W}\rangle$ arises from the presence of a parallel electric field; when infinite plasma conductivity parallel to the B-lines is assumed, precluding the existence of $E_{\|}$, 〈W drops one order in $\epsilon$. We next further restrict the problem by assuming that the only source of magnetic fields is an arbitrarily shaped conducting magnet whose motion conserves the particle's magnetic moment $\mathrm{M}$ and second invariant $\mathrm{J}$, but is otherwise arbitrary. The pattern of currents in the magnet's frame is assumed invariant, so that the magnetic field moves rigidly. In this case energy changes $\langle\dot{W}\rangle$ occur on a still slower time scale than when $E_{\|}=0$ alone.

Our principal conclusion is that the slow variation of $\langle\dot{W}\rangle$ found by Hones and $\underline{\text { Bergeson for }} \mathrm{E}_{\|}=0$ and a dipole magnetic source is a particular example of a theorem applicable to general motions of arbitrarily shaped magnets.

\section{A GENERAL EXPRESSION FOR 〈ì}

The general representation of an electric field $\mathbf{E}(\mathbf{r}, t)$ in terms of the electrostatic potential $\phi(\mathbf{r}, t)$ and the magnetic vector potential $\mathbf{A}(\mathbf{r}, t)$ is

$$
\mathbf{E}=-\nabla \phi-\frac{1}{c} \frac{\partial \mathbf{A}}{\partial t} .
$$


An alternative form of $\mathbf{A}$ is

$$
\mathbf{A}(\mathbf{r}, \mathrm{t})=\alpha(\mathbf{r}, \mathrm{t}) \boldsymbol{\nabla} \beta(\mathbf{r}, \mathrm{t})+\boldsymbol{\nabla} \tau(\alpha, \beta, \mathrm{t}) .
$$

In Equation (2) $\alpha$ and $\beta$ are functions of space and time such that magnetic field lines are the intersections of surfaces $\alpha=$ const. with surfaces of $\beta=$ const. The magnetic field $\mathbf{B}$ is given in magnitude and direction by

$$
\mathbf{B}(\mathbf{r}, \mathrm{t})=\boldsymbol{\nabla} \alpha(\mathbf{r}, \mathrm{t}) \times \boldsymbol{\nabla} \beta(\mathbf{r}, \mathrm{t})
$$

The fact that there exists an infinity of pairs $\alpha-\beta$ for a given $B$ is of no concern, for the resultant expression for $\langle\dot{w}\rangle$ is independent of the choice of a particular $\alpha-\beta$ pair. Given an $\alpha-\beta$ pair, $\nabla \tau$ in Equation (2) represents this gauge freedom, and since $\langle\dot{W}\rangle$ is gauge invariant we set $\tau=0$.

Once functions $\alpha$ and $\beta$ are chosen, it is readily shown [Northrop, 1963] that each magnetic field line retains its $\alpha, \beta$ labels as it moves in time through space with the field line velocity

$$
\mathbf{v}_{\ell}=\left(\frac{\partial \beta}{\partial \mathrm{t}} \nabla \alpha-\frac{\partial \alpha}{\partial \mathrm{t}} \nabla \beta\right) \times \frac{\mathbf{B}}{\mathrm{B}^{2}} .
$$

A point $\mathbf{r}$ in space is labeled at time $\mathbf{t}$ by the $\alpha-\beta$ values of the magnetic field line presently passing through it, together with a measure of arc length $s(\mathbf{r}, t)$ measured along the field line from an arbitrary reference surface. The set of independent variables $\alpha, \beta, \mathrm{s}, \mathrm{t}$ can thus be used instead of the set $\mathbf{r}, \mathrm{t}$. 
With this introduction to $\alpha, \beta$, and $s$ in hand, Equation (1) becomes

$$
\mathbf{E}=-\nabla(\phi+\psi)+\frac{1}{\mathrm{c}}\left[\frac{\partial \beta}{\partial \mathrm{t}} \nabla \alpha-\frac{\partial \alpha}{\partial \mathrm{t}} \nabla \beta\right],
$$

with $\psi$ defined as

$$
\psi=\frac{1}{c} a \frac{\partial \beta(\mathbf{r}, \mathbf{t})}{\partial t} .
$$

Since $\alpha$ and $\beta$ are constant on field lines, $\nabla \alpha$ and $\nabla \beta$ lie perpendicular to field lines, and

$$
\mathbf{E}_{\|}=\frac{\mathbf{E} \cdot \mathbf{B}}{\mathbf{B}}=-\frac{\mathbf{B}}{\mathbf{B}} \cdot \nabla(\phi+\psi)=-\frac{\partial}{\partial \mathbf{s}}(\phi+\psi) .
$$

$\phi+\psi$ is thus a potential for the parallel electric field and is constant on a field line segment where $\mathrm{E}_{\|}=0$.

In adiabatic theory there is associated with each particle the useful quantity

$$
\mathrm{K}=\mathrm{W}+\mathrm{e}(\phi+\psi)
$$

where $W$ is the particle kinetic energy and $e(\phi+\psi)$ is evaluated at the particle position. For static fields $\psi=0$ and $\mathrm{K}$ is the total energy of the particle moving in the potential $\phi$. 
From Equation (8) we determine the instantaneous time rate of change of particle kinetic energy to be

$$
\dot{\mathbf{W}}=\dot{\mathrm{K}}-\mathbf{e}(\dot{\phi}+\dot{\psi}) \text {, }
$$

a dot denoting a time variation as seen by the moving particle.

Let us denote the average of the arbitrary quantity $a(t)$ over the bounce period $\mathrm{T}$ of a given particle by $\langle\mathrm{a}\rangle$,

$$
\langle a\rangle=T^{-1} \int_{t}^{t+T} d t^{\prime} a\left(t^{\prime}\right)=\left[\oint \frac{d s}{v_{\|}}\right]^{-1} \oint \frac{d s}{v_{\|}} a,
$$

the s integrals extending over a complete guiding center bounce path, which in second adiabatic motion very closely follows a magnetic field line. Averaging Equation (9) in this way, we obtain for the bounce averaged rate of change of particle energy

$$
\langle\dot{W}\rangle=\langle\dot{\mathrm{K}}\rangle-\mathrm{e}[\dot{\phi}\rangle+\langle\dot{\psi}\rangle] .
$$

Northrop [1963] has shown

$$
\frac{1}{\mathrm{e}}\langle\dot{\mathrm{K}}\rangle=-\frac{1}{\mathrm{eT}} \frac{\partial \mathrm{J}(\alpha, \beta, \mathrm{K}, \mathrm{M}, \mathrm{t})}{\partial \mathrm{t}}+\Theta\left(\epsilon^{3}\right)
$$


where $\mathrm{J}$ is the particle longitudinal invariant defined, using Equation (8), as a function of $\alpha, \beta, \mathrm{K}, \mathrm{M}\left(=\mathrm{W}_{1} / \mathrm{B}\right)$, and $\mathrm{t}$ as

$$
\begin{aligned}
& J(\alpha, \beta, K, M, t)=m \oint v_{\|} d s \\
& =(2 m)^{1 / 2} \oint[K-e \Sigma(\alpha, \beta, s, t)-M B(\alpha, \beta, s, t)]^{1 / 2} d s .
\end{aligned}
$$

In Equation (13) $\sum$ and $B$ are respectively $[\phi+\psi](\mathbf{r}, t)$ and $B(\mathbf{r}, t)$ expressed in terms of the variables $\alpha, \beta, s$, and $t$. The $\Theta\left(\epsilon^{3}\right)$ in Equation (12) indicates that terms neglected are asymptotically of third order in the smallness parameter $\epsilon$. Since both $\mathrm{K}$ and $\mathrm{J}$ contain a factor $\mathrm{m}, 1$ /e appears on both sides of Equation (12) simply for ordering purposes.

Taking $\partial / \partial \mathrm{t}$ of Equation (13) (at constant $\alpha, \beta, \mathrm{K}$, and $\mathrm{M}$ ), we obtain

$$
\frac{\partial J}{\partial t}=-T\left[e\left\langle\frac{\partial \Sigma}{\partial t}\right\rangle+M\left\langle\frac{\partial B}{\partial t}\right\rangle\right] \text {. }
$$

The expression for $\langle\dot{W}\rangle / \mathrm{e}$,

$$
\frac{\langle\dot{W}\rangle}{\mathrm{e}}=\left\langle\frac{\partial \Sigma}{\partial \mathrm{t}}\right\rangle+\frac{\mathrm{M}}{\mathrm{e}}\left\langle\frac{\partial B}{\partial \mathrm{t}}\right\rangle-\langle\dot{\Sigma}\rangle+\theta\left(\epsilon^{3}\right),
$$


follows by combining Equations (11), (12), and (14). Noting that

$$
\langle\dot{\phi}(\mathbf{r}, \mathbf{t})+\dot{\psi}(\mathbf{r}, \mathbf{t})\rangle=\langle\dot{\Sigma}(\alpha, \beta, \mathbf{s}, \mathbf{t})\rangle=\left\langle\frac{\partial \Sigma}{\partial t}+\dot{\alpha} \frac{\partial \Sigma}{\partial \alpha}+\dot{\beta} \frac{\partial \Sigma}{\partial \beta}+\mathbf{v}_{\|} \frac{\partial \Sigma}{\partial \mathbf{s}}\right\rangle
$$

we alternatively write Equation (15) in the form

$$
\frac{\langle\dot{w}\rangle}{\mathrm{e}}=\frac{\mathrm{M}}{\mathrm{e}}\left\langle\frac{\partial B}{\partial \mathrm{t}}\right\rangle-\left\langle\dot{\alpha} \frac{\partial \Sigma}{\partial \alpha}+\dot{\beta} \frac{\partial \Sigma}{\partial \beta}+\mathrm{v}_{\|} \frac{\partial \Sigma}{\partial \mathrm{s}}\right\rangle+\theta\left(\epsilon^{3}\right)
$$

where in terms of the guiding center drift velocity $\mathbf{v}_{\mathbf{D}}$ and the field line velocity $\mathbf{v}_{\ell}, \dot{\alpha}$ and $\dot{\beta}$ are $\left(\mathbf{v}_{\mathbf{D}}-\mathbf{v}_{\ell}\right) \cdot \nabla \alpha$ and $\left(\mathbf{v}_{\mathbf{D}}-\mathbf{v}_{\ell}\right) \cdot \nabla \beta$ respectively.

Requisites for the existence of the invariants $M$ and $J$ are that time variations of $\mathrm{B}$ take place on the guiding center drift time scale (an $\epsilon^{-1}$ time scale) and that $\mathbf{E}$ be of order $\epsilon$. Under these conditions $\partial B / \partial t, \dot{\alpha}, \dot{\beta}$, and $\Sigma$ are $Q(\epsilon)$ quantities, and the leading order contribution to Equation (17) comes from the $\mathbf{E}_{\| !}$(i.e. $\partial \Sigma / \partial \mathrm{s}$ ) term. If $\mathrm{E}_{\|} \neq 0$, we find using Equation $(10)$

$$
\frac{\langle\dot{W}\rangle}{\mathrm{e}}=-\frac{2}{\mathrm{~T}}\left\{\Sigma\left(\alpha, \beta, \mathrm{s}_{\mathrm{u}}, \mathrm{t}\right)-\Sigma\left(\alpha, \beta, \mathrm{s}_{\ell}, \mathrm{t}\right)\right\}+\theta\left(\epsilon^{2}\right)
$$

where $s_{u}$ and $s_{\ell}$ are the upper and lower mirror points for a particle with invariants $\mathrm{J}$ and $\mathrm{M}$ whose guiding center is on the line $\alpha, \beta$. Even a small amount of $\mathbf{E}_{\|}$can thus produce significant energy changes on the drift time scale. 


\section{$\langle\dot{W}\rangle$ IN SPECIAL CASES}

For $\mathbf{E}_{\|}=0, \Sigma$ is independent of $\mathbf{s}$ as shown in the preceding section and Equation (17) reduces to

$$
\frac{\langle\dot{w}\rangle}{\mathrm{e}}=\frac{\mathrm{M}}{\mathrm{e}}\left\langle\frac{\partial B}{\partial \mathrm{t}}\right\rangle-\langle\dot{a}\rangle \frac{\partial \Sigma}{\partial \alpha}-\langle\dot{\beta}\rangle \frac{\partial \Sigma}{\partial \beta}+\sigma\left(\epsilon^{3}\right) .
$$

From the relationships

$$
\begin{aligned}
& \langle\dot{\alpha}\rangle=-\frac{\mathrm{c}}{\mathrm{e}} \frac{\partial \mathrm{K}(\alpha, \beta, \mathrm{J}, \mathrm{M}, \mathrm{t})}{\partial \beta}=\frac{\mathrm{c}}{\mathrm{eT}} \frac{\partial \mathrm{J}(\alpha, \beta, \mathrm{K}, \mathrm{M}, \mathrm{t})}{\partial \beta}, \\
& \langle\dot{\beta}\rangle=\frac{\mathrm{c}}{\mathrm{e}} \frac{\partial \mathrm{K}(\alpha, \beta, \mathrm{J}, \mathrm{M}, \mathrm{t})}{\partial \alpha}=-\frac{\mathrm{c}}{\mathrm{eT}} \frac{\partial \mathrm{J}(\alpha, \beta, \mathrm{K}, \mathrm{M}, \mathrm{t})}{\partial \beta},
\end{aligned}
$$

derived by Northrop [1963], there follow the alternative forms of Equation (19):

$$
\begin{aligned}
& \frac{\langle\dot{W}\rangle}{\mathrm{e}}=\frac{\mathrm{M}}{\mathrm{e}}\left\langle\frac{\partial B}{\partial \mathrm{t}}\right\rangle+\frac{\mathrm{c}}{\mathrm{e}} *\left(\frac{\mathrm{K}, \Sigma}{\beta, a}\right)+\theta\left(\epsilon^{3}\right), \\
& \frac{\langle\dot{W}\rangle}{\mathrm{e}}=\frac{\mathrm{M}}{\mathrm{e}}\left\langle\frac{\partial B}{\partial \mathrm{t}}\right\rangle-\frac{\mathrm{c}}{\mathrm{e}} *\left(\frac{\mathrm{J}, \Sigma}{\beta, a}\right)+\Theta\left(\epsilon^{3}\right),
\end{aligned}
$$

\& being the Jacobian operator. All terms in the forms (19-21) are now $Q\left(\epsilon^{2}\right)$ and we conclude that the absence of $E_{\|}$slows the energy change by one order.

When the only source of magnetic fields is a moving conducting magnet, and the magnetic field moves rigidly with the magnet, $\partial B / \partial t(\alpha, \beta, s, t)=0$ : to an observer moving so as to always remain at point $s$ on the field line $\alpha, \beta$, no magnetic field changes are apparent. Also, since $\Sigma$ is independent of $s$, it is 
the same at any exterior point on a field line as at the point where the line enters the magnet. Therefore, if $\Sigma$ is known everywhere on the surface of the magnet, $\langle\dot{W}\rangle$ can be obtained at all exterior points from any of the forms (19-21). The problem is reduced to determining $\Sigma$ on the surface of the magnet.

Inside the moving conducting magnet we assume that conduction electrons are in equilibrium, i.e.

$$
\mathbf{E}+\frac{\mathbf{v}(\mathbf{r}, \mathrm{t}) \times \mathbf{B}}{\mathrm{c}}=-\boldsymbol{\nabla} \phi-\frac{1}{\mathrm{c}} \frac{\partial \mathbf{A}}{\partial \mathrm{t}}+\frac{\mathbf{v} \times(\boldsymbol{\nabla} \times \mathbf{A})}{\mathrm{c}}=0
$$

where $\mathbf{v}(\mathbf{r}, t)$ is the velocity of a point in the magnet located at $\mathbf{r}$ in the inertial frame ("laboratory frame") in which $\langle\dot{W}\rangle$ is to be evaluated. It will be further assumed that only the permanent magnetization contributes to $\mathbf{A}$, which then moves rigidly with the magnet. The following considerations follow closely those of Backus [1956] but allow for arbitrary motion of the conducting magnet. Vector manipulation of Equation (22) leads to

$$
\boldsymbol{\nabla} \phi+\frac{1}{\mathrm{c}} \frac{\partial \mathbf{A}}{\partial \mathrm{t}}=\frac{1}{\mathrm{c}}[\boldsymbol{\nabla}(\mathbf{v} \cdot \mathbf{A})-\mathbf{A} \cdot \boldsymbol{\nabla v}-\mathbf{A} \times(\boldsymbol{\nabla} \times \mathbf{v})-\mathbf{v} \cdot \nabla \mathbf{A}] .
$$

Here $\mathbf{A}, \phi, \mathbf{v}$, and $\mathbf{r}$ are all measured in the laboratory frame; $\partial \mathbf{A} / \partial t$, the rate of change of A seen by an observer fixed in the laboratory frame at $\mathbf{r}$, arises from both rotation and translation of the magnet:

$$
\frac{\partial \mathbf{A}}{\partial \mathrm{t}}=\Omega(\mathrm{t}) \times \mathbf{A}-\mathbf{v} \cdot \boldsymbol{\nabla A}
$$


The instantaneous angular velocity $\Omega$ is defined in terms of the instantaneous rotation rate with respect to the laboratory coordinates of any set of axes fixed in the conducting body. Further,

$$
\mathbf{v}=\mathbf{v}_{0}(t)+\Omega(t) \times\left[\mathbf{r}-\mathbf{r}_{0}(t)\right]
$$

where $\mathbf{v}_{0}$ and $\mathbf{r}_{0}$ are the velocity and displacement of the origin of the body axes with respect to the origin of laboratory coordinates. Substituting from Equations (24) and (25) into Equation (23), we obtain after performing the $\boldsymbol{\nabla}$-operations

$$
\nabla\left(\phi-\frac{\mathbf{v} \cdot \mathbf{A}}{c}\right)=0
$$

This result is identical with that of Backus, who considered the special case where the axis of rotation is fixed in the laboratory frame.

Thus, interior to the conducting magnet

$$
\phi=\frac{\mathbf{v} \cdot \mathbf{A}}{\mathrm{c}}+\mathrm{D}(\mathrm{t})
$$

where $D(t)$ is arbitrary, so that in this region

$$
\begin{aligned}
\phi+\psi & =+\frac{\mathbf{v} \cdot \mathbf{A}}{\mathrm{c}}+\frac{1}{\mathrm{c}} a \frac{\partial \beta}{\partial \mathrm{t}}+\mathrm{D}(\mathrm{t}) \\
& =\frac{a}{\mathrm{c}}\left[\frac{\partial \beta}{\partial \mathrm{t}}+\mathbf{v} \cdot \nabla \beta\right]+\mathrm{D}(\mathrm{t}) .
\end{aligned}
$$


Since the field moves rigidly with the magnet, $\partial \beta / \partial t+\mathbf{v} \cdot \nabla \beta \equiv 0$ and $\phi+\psi$ is simply an arbitrary function of time.

Taking for boundary conditions the continuity of $\phi$ and $\psi$, we conclude that $\phi+\psi$ is independent of spatial position outside the magnet, since all exterior points are connected to the magnet by magnetic field lines and $\phi+\psi$ is s-independent. It then follows that $\partial \Sigma / \partial a=0, \partial \Sigma / \partial \beta=0$, and with $\partial B / \partial t=0$, Equation (19) predicts

$$
\frac{\langle i \omega}{e}=0+\theta\left(\epsilon^{3}\right) \text {. }
$$

Thus the average rate of energy change drops by two orders in $\epsilon$.

\section{CONCLUDING REMARKS}

We would emphasize that the principal result of this paper-that the bounce averaged rate of energy change of trapped particles is of $Q\left(\epsilon^{3}\right)$ small in the adiabatic parameter-is a theorem, proved for the idealized model in which $\mathbf{E}_{\|}=0$ and $\mathbf{B}$ arises solely from and moves rigidly with a conducting magnet. If the entire magnetic field surrounding the earth were due to the earth's internal currents (and $E_{\|}=0$ ) the wobbling field caused by the earth's rotation would not affect trapped particle energies. The existence in reality, however, of small finite parallel resistivity and concommitant $E_{\|}$and the presence of nonrigidly convected magnet field sources-e.g. ring, magnetopause, and tail currents-push us into the range of validity of Equations (18 and 20) and their predictions of much more rapid energy changes. 


\section{REFERENCES}

Backus, G., The external electric field of a rotating magnet, Astrophys. J., 123 $508,1956$.

Davis, L. Jr., Stellar electromagnetic fields, Phys. Rev., 72, 632, 1947.

Davis, L.Jr., Stellar electromagnetic fields, Phys. Rev., 73, 536, 1948.

Hones, E. W. Jr., and J. E. Bergeson, Electric field generated by a rotating magnetized sphere, J. Geophys。 Res., 70, 4951, 1965.

Northrop, T. G., The Adiabatic Motion of Charged Particles, John Wiley and Sons (Interscience), New York, 1963.

Terletzky, J., Induction of fast charged particle currents by rotating magnetized cosmic bodies, J. Phys. (Russian), 10(4), 377, 1946. 